\title{
Low Unconguted Estriol Levels in the Maternal Quadruple-Marker Screen in a Fetus with Cornelia de Lange Syndrome and a Partial Deletion of the NIPBL Gene
}

\author{
Patrick L. Wilson ${ }^{1, *}$, Jean Ricci Goodman ${ }^{1,3}$, Erica Cole ${ }^{2}$, John J. Mulvihill ${ }^{2}$, Ji-Yun Lee ${ }^{2,3,4}$, Shibo Li ${ }^{2}$, \\ Andrew Wagner ${ }^{1}$ \\ ${ }^{1}$ Department of Obstetrics and Gynecology, Maternal/Fetal Medicine, University of Oklahoma Health Sciences Center, Oklahoma City, \\ OK, USA \\ ${ }^{2}$ Department of Pediatrics, Genetics Section, University of Oklahoma Health Sciences Center, Oklahoma City, OK, USA \\ ${ }^{3}$ Deparment of Obstetrics and Gynecology, Maternal/Fetal Medicine, Loyola University, Stritch School of Medicine, Chicago, IL, USA \\ ${ }^{4}$ Department of Pathology, Korea University, Seoul, Korea \\ *Corresponding author: Patrick-L-Wilson@ ouhsc.edu
}

Received December 29, 2012; Revised February 04, 2013; Accepted March 15, 2013

\begin{abstract}
Prenatal diagnosis of Cornelia de Lange syndrome (CDLs) is difficult due to non-specific ultrasound and biochemical findings, normal karyotype, and negative family history. Previous studies suggest that low first and second trimester levels of pregnancy associated plasma protein-A are an indicator for this genetically heterogeneous condition, low levels of this protein can be non-specific. Here, we suggest the addition of estriol to the prenatal evaluation of CdLs. This steroid hormone, produced by the placenta from fetal 16-hydroxydehydroepiandrosterone, can serve as an indicator for fetal distress, placental dysfunction, and intrauterine growth retardation which is a feature of CdLs.
\end{abstract}

Keywords: Cornelia de Lange Syndrome, prenatal, quadruple screen, trisomy 18, microarray, Smith Lemli Opitz syndrome, placenta

\section{Introduction}

Cornelia de Lange syndrome, type 1 (CdLs), is an autosomal dominant genetic condition with a frequency that may be as high as 1 in 10,000 live births. This multisystem condition is most commonly associated with mutations in the NIPBL gene on chromosome $5 \mathrm{p} 13.1$. This human homolog of the Drosophila Nipped-B gene is active in regulating sister chromatid cohesion and other proteins that function in the Notch signaling pathway. The severity of CdLs is associated with the presence and type of mutation: nonsense, missense, frameshift, or splicing. Phenotypically, CdLs can have a varied presentation. Craniofacial features include microbrachycephaly, arched and long eyebrows with synophrys, long eyelashes, lowset ears, small upturned nose, long philtrum, thin lips, downturned corners of the mouth, microretrognathia, and low-set, posteriorly rotated ears. Upper-limb malformations vary from subtle changes in the phalanges and metacarpal bones and small hands to oligodactyly and severe reduction defects. Additional clinical features include hearing loss, ptosis, myopia, cryptorchidism, hypospadias, cardiac septal defects, cognitive and growth retardation, gastrointestinal abnormalities, and diaphragmatic hernia $[1,2]$.
Here we present a case of CdLs in which the second trimester multiple marker screening test showed an increased risk for trisomy 18, 1 in 11. Ultrasound findings included micrognathia, overlapping fingers on the right hand, bilateral cleft feet with hyperextended 1 st toes, and growth restriction. These along with low maternal serum estriol levels raised the concern for a fetal abnormality, e.g. Smith-Lemli-Opitz syndrome or X-linked ichthyosis. After consulting with the patient, an amniocentesis was performed. Fetal karyotype was normal; however, postnatal diagnosis of CdLs was determined by whole genome oligonucleotide microarray analysis that revealed an approximately $312 \mathrm{~kb}$ loss on the short arm of chromosome 5p13.2 involving the NIPBL, IDN3, and NUP155 genes (Figure 1).

\section{Case Presentation}

A 26yo $\mathrm{G}_{3} \mathrm{P}_{1102}$ Caucasian female was referred due to an increased risk for trisomy 18 (1:11) on a maternal quadruple marker screen at $18-6 / 7$ weeks. Unconjugated estriol (uE3) levels were decreased at $0.30 \mathrm{ng} / \mathrm{mL}, 0.30$ MoM. The initial ultrasound did not reveal any fetal abnormalities but was limited by maternal body habitus and fetal positioning. Our patient returned 4 weeks later. Micrognathia, overlapping fingers on the right hand, 
bilateral cleft feet with hyperextended 1st toes, and growth restriction were seen. At this time, she underwent amniocentesis that revealed a 46,XY karyotype. DHCR7 gene sequencing for Smith-Lemli-Opitz syndrome due to the low uE3 level was normal. At 31 weeks she was hospitalized due to IUGR and had a repeat $\mathrm{C} / \mathrm{S}$ due to a non-reassuring biophysical profile.
Postnatal diagnosis of CdLs was determined by whole genome oligonucleotide microarray analysis that revealed an approximately $312 \mathrm{~kb}$ loss of the short arm of chromosome 5 at band p13.2 involving the NIPBL, IDN3, and NUP155 genes (Figure 1).

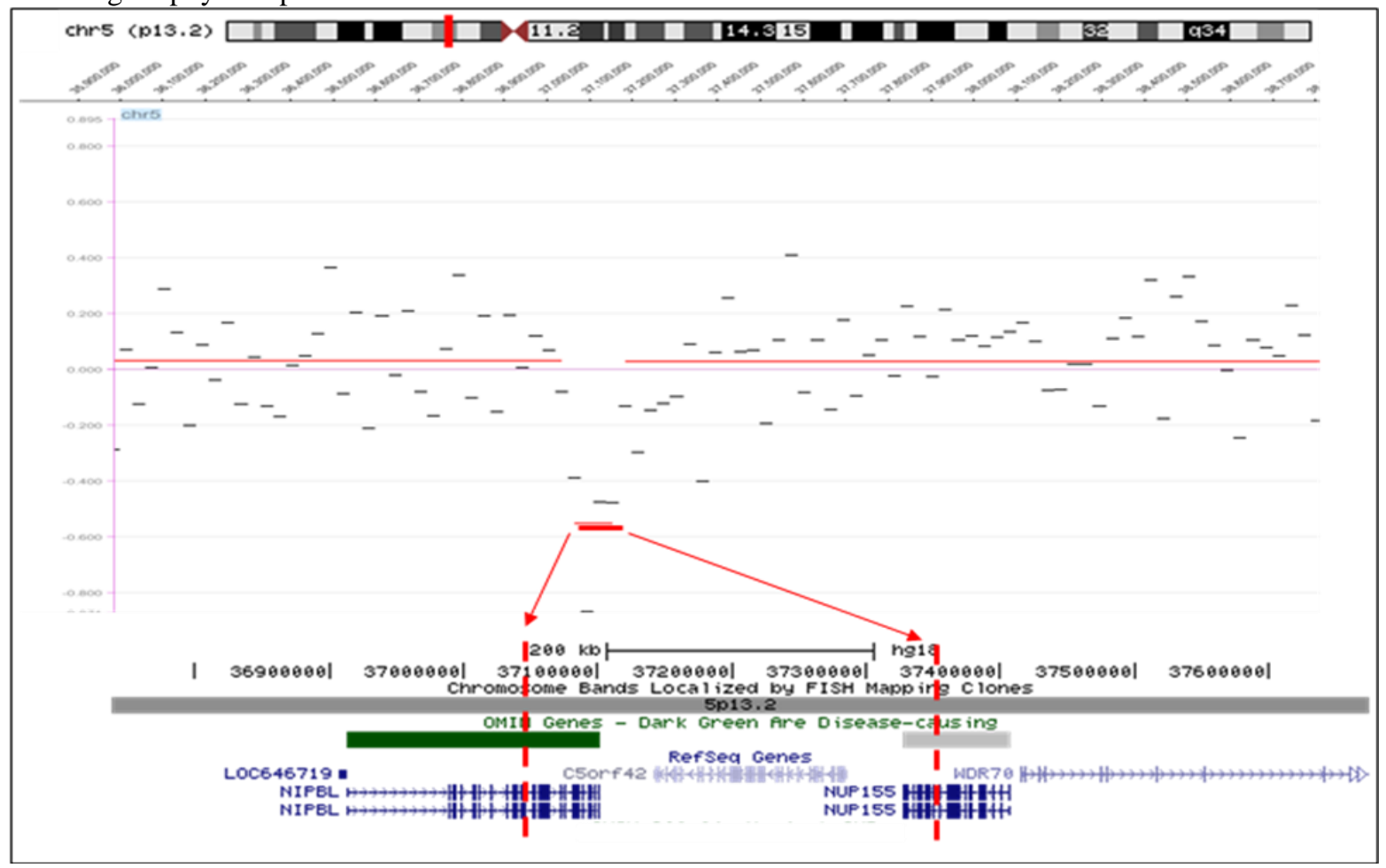

Figure 1. Whole genome oligonucleotide microarray analysis revealed a loss on the short arm of chromosome 5 involving band p13.2. This loss is approximately $312 \mathrm{~kb}$ in size $(37,050,251 \mathrm{bp} \rightarrow 37,362,606 \mathrm{bp})$ and includes 3 genes (NIPBL, IDN3, and NUP155). Defects in NIPBL are the cause of Cornelia de Lange syndrome type 1 (CDLS1). (385kb oligonucleotide array, Roche NimbleGen System Inc. (Madison, WI), UCSC Genome Browser on Human Mar. 2006 (NCBI36/hg18) Assembly)

Results from the genetics evaluation included a pheontypically male infant with an annular pancreas causing duodenal stenosis, a generous posterior fontanelle, synophrys, long eyelashes, micrognathia, cleft palate, hypospadias, unilateral cryptorchidism, left single palmar crease, absent right second finger, bilateral cleft feet with incomplete development of toes on the right foot. He underwent exploratory laparotomy and gastroduodenostomy due to a partial rotational anomaly with emergent tracheostomy at 33 days and expired at 37 days.

Maternal karyotype was 46,XX, and microarray analysis was negative for the $5 \mathrm{p} 13.2$ microdeletion. The baby's father was unavailable for testing.

\section{Discussion}

Prenatal ultrasound findings of growth retardation, limb defects, diaphragmatic hernia, and abnormal facial features are common in CdLs. However, due to the variability in the CdLs phenotype these features may not be present in affected fetuses. Similarly, low first and second trimester maternal serum pregnancy-associated plasma protein A (PAPP-A) have been associated with CdLs. The difficulty here is that PAPP-A measurements must be taken over a period of time, and PAPP-A is not a fetal analyte that is routinely measured in maternal serum during second trimester pregnancy screening tests $[3,4,5]$.

Second trimester maternal serum screening occurs between weeks 15-20 of the pregnancy. The gravid woman submits a blood sample for analysis. Alphafetoprotein (AFP), unconjugated estriol $\left(\mathrm{uE}_{3}\right)$, and human chorionic gonadotropin (hCG) in maternal blood along with maternal demographic factors (age, weight, gestational age, race, diabetic status) are used in the determination of the fetus' risk to have trisomy 18, Down syndrome, or an open neural tube/abdominal wall defect. Using a false positive rate of $5 \%$, the "triple screen" has a sensitivity of $\sim 70 \%$ for trisomy 18 [6].

Estriol, one of three naturally occurring estrogens, is typically highest during a woman's childbearing years and increases during pregnancy. Its production depends on an intact maternal-fetal-placental unit. Fetal adrenal glands produce dehydroepiandrosterone. The placenta converts 16-hydroxy-dehydroepiandrosterone sulfate to androgens, which are aromatized to estriol. Estriol then diffuses through the placenta into the mother's blood system where it can be measured as unconjugated estriol $[7,8]$.

The production of $\mathrm{uE}_{3}$ can be evaluated through its progressive rise in maternal circulation reaching a peak late in gestation that is several orders of magnitude greater than non-pregnant levels. The combined evaluation of maternal serum AFP, hCG, $\mathrm{uE}_{3}$, and maternal age have value in predicting risk for fetal chromosomal 
abnormalities during pregnancy. The use of maternal serum AFP, hCG, and $\mathrm{uE}_{3}$ predicts $70 \%$ of fetuses diagnosed with trisomy 18 . Low levels of $\mathrm{uE}_{3}$, less than or equal to $0.5 \mathrm{MoM}$, have been associated with adverse pregnancy outcomes: IUGR and early fetal loss. Levels less than $0.25 \mathrm{MoM}$ are indicators that the fetus might be experiencing congenital adrenal hypoplasia, steroid sulfates deficiency, or Smith-Lemli-Opitz syndrome [8,9].

During a normal pregnancy, the levels of AFP, hCG, and $\mathrm{uE}_{3}$ are never simultaneously low. This is seen in less than $2 \%$ of all maternal serum screens performed during the second trimester. Of these, only $4-6 \%$ of fetuses will have trisomy 18 [10]. Our recent experience suggests that Cornelia de Lange syndrome be included in the differential diagnosis when the multiple marker screen is positive for trisomy 18 .

\section{References}

[1] Gillis, L.A., McCallum, J., Kaur, M., DeScipio, C., Yaeger, D. Mariani, A., Kline, A.D., Li, H.H., Devoto, M., Jackson, L.G., and Krantz I.D, "NIPBL mutational analysis in 120 individuals with Cornelia de Lange syndrome and evaluation of genotypephenotype correlations," American Journal of Human Genetics, 75 (4). 610-23. Oct.2004.

[2] Lalatta, F., Russo, S., Gentilin, B., Spaccini, L., Boschetto, C., Cavalleri, F., Masciadri, M., Gervasini, C., Bentivegna, A., Castronovo, P., and Larizza, L, "Prenatal/neonatal pathology in two cases of Cornelia de Lange syndrome harboring novel mutations of NIPBL," Genetics in Medicine, 9 (3). 188-94. Mar.2007.
[3] Westergaard, J.G., Chemnitz, J., Teisner, B., Poulsen, H.K., Ipsen, L., Beck, B., and Grudzinskas, J,G, "Pregnancy-associated plasma protein A: a possible marker in the classification and prenatal diagnosis of Cornelia de Lange syndrome," Prenatal Diagnosis, 3(3). 225-32. Jul.1983.

[4] Aitken, D.A., Ireland, M., Berry, E., Crossley, J.A., Macri, J.N., Burn, J., and Connor J,M, "Second-trimester pregnancy associated plasma protein-A levels are reduced in Cornelia de Lange syndrome pregnancies," Prenatal Diagnosis, 19(8). 706-10. Aug.1999.

[5] Arbuzova. S., Nikolenko. M., Krantz. D., Hallahan. T., and Macri, $\mathrm{J}$, "Low first trimester pregnancy-associated plasma protein-A and Cornelia de Lange syndrome," Prenatal Diagnosis, 23(10). 864. Oct.2003.

[6] Driscoll, D.A. and Gross, S.J, "Screening for fetal aneuploidy and neural tube defects," Genetics in Medicine, 11 (11). 818-21. Nov.2009.

[7] Schleifer, R.A., Bradley, L.A., Richards, D.S., and Ponting, N.R, "Pregnancy outcome for women with very low levels of maternal serum unconjugated estriol on second-trimester screening," American Journal of Obstetrics and Gynecology, 173(4) .1152-6. Oct.1995.

[8] Kim, S.Y., Kim, S.K., Lee, J.S., Kim, I.K., and Lee, K, “The prediction of adverse pregnancy outcome using low unconjugated estriol in the second trimester of pregnancy without risk of Down's syndrome," Yonsei Medical Journal, 41(2). 226-9. Apr.2000.

[9] Dugoff, L, Society For Maternal-Fetal Medicine, "First- and second-trimester maternal serum markers for aneuploidy and adverse obstetric outcomes," Obstetrics and Gynecology, 115(5). 1052-61. May.2010.

[10] Wenstrom, K.D,, Owen, J., Brumfield, C.G., Davis, R.O., Dubard, M., and Garcia T, "Significance of a false-positive trisomy 18 multiple-marker screening test," Obstetrics and Gynecology, 90(6). 938-42. Dec. 1997. 